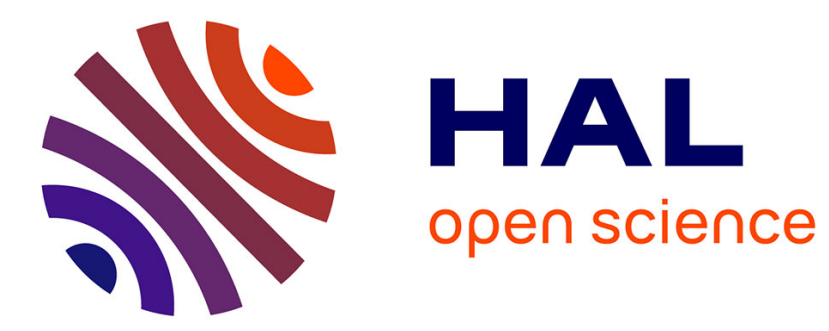

\title{
Stretching of a polymer by an attractive wall
}

\author{
A. Johner, E. Bouchaud, M. Daoud
}

\section{To cite this version:}

A. Johner, E. Bouchaud, M. Daoud. Stretching of a polymer by an attractive wall. Journal de Physique, 1990, 51 (6), pp.495-502. 10.1051/jphys:01990005106049500 . jpa-00212385

\section{HAL Id: jpa-00212385 https://hal.science/jpa-00212385}

Submitted on 1 Jan 1990

HAL is a multi-disciplinary open access archive for the deposit and dissemination of scientific research documents, whether they are published or not. The documents may come from teaching and research institutions in France or abroad, or from public or private research centers.
L'archive ouverte pluridisciplinaire HAL, est destinée au dépôt et à la diffusion de documents scientifiques de niveau recherche, publiés ou non, émanant des établissements d'enseignement et de recherche français ou étrangers, des laboratoires publics ou privés. 


\title{
LE JOURNAL DE PHYSIQUE
}

J. Phys. France 51 (1990) 495-502

15 MARS 1990, PAGE 495

Classification

Physics Abstracts

$36.20 \mathrm{E}-68.10 \mathrm{~J}$

\section{Short Communication}

\section{Stretching of a polymer by an attractive wall}

\author{
A. Johner $\left({ }^{1}\right)$, E. Bouchaud $\left({ }^{2}\right)$ and M. Daoud $\left({ }^{3}\right)$ \\ ( ${ }^{1}$ I.C.S.-C.R.M., 6 rue Boussingault, 67083 Strasbourg Cedex, France \\ $\left({ }^{2}\right)$ O.N.E.R.A., B.P. 72, 92322 Chatillon Cedex, France \\ $\left({ }^{3}\right)$ Laboratoire Léon Brillouin $\left({ }^{*}\right)$, C.E.N. Saclay, 91191 Gif-sur-Yvette Cedex, France
}

(Reçu le 24 novembre 1989, accepté sous forme définitive le 15 janvier 1990)

Résumé. - En utilisant des arguments d'échelle, nous généralisons au cas de polymères à volume exclu un récent calcul exact de champ moyen des configurations d'une chaîne dont une extremité est fixée à une distance $z$ d'un plan adsorbant. En plus de l'épaisseur caractéristique du polymère adsorbe, il existe une seconde longueur qui a été ignorée dans les discussions récentes. Elle correspond à une distance de capture $Z_{0}$ à partir de laquelle la chaîne commence à être attirée par la surface : pour des distances plus grandes, le polymère ne sent pas la surface. Pour des distances plus petites, on doit appliquer une force constante à l'extrémité pour la maintenir fixe, et la chaîne adopte une configuration étirée. Nous trouvons $Z_{0} \sim N \delta^{2 / 3}$, où $N$ est la longueur de la chaîne et $\delta$ le gain d'énergie par monomère sur la surface. Nous étudions également la relaxation d'une telle chaîne étirée lorsque la contrainte sur l'extrémité fixe est supprimée. Elle relaxe par un mouvement de succion par la surface. Nous trouvons un temps caracteristique $T \sim N^{2} \delta^{1 / 3}$ pour ce dernier processus. Ceci peut être interessant pour l'arrachement d'une chaine adsorbée. Notre approche se fonde sur celle de Pincus pour une chaîne étirée.

Abstract . - Using scaling arguments, we generalize to the excluded volume case an exact calculation that was made in a mean field calculation for the configuration of a polymer chain with one end fixed at a distance $z$ from an adsorbing plane. In addition to the characteristic width of the adsorbed chain, there is a second distance that was ignored so far in subsequent discussions. This is the capture distance $Z_{0}$ when the polymer starts being attracted by the surface: for larger distances the chain does not feel the surface. For smaller distances, if one end of the chain is fixed, a constant force has to be applied to keep it fixed and the polymer adopts a stretched configuration. We find $Z_{0} \sim N \delta^{2 / 3}$, where $N$ is the length of the chain and $\delta$ the energy gain per monomer on the surface. We proceed by looking at the relaxation of such elongated chain when the constraint of fixed end is released. In this process, the chain relaxes the applied force by being sucked onto the surface. We find for the

$\left(^{*}\right)$ Laboratoire commun C.N.R.S.-C.E.A. 
characteristic time for this process $T \sim N^{2} \delta^{1 / 3}$. This may be relevant for extracting a chain from an adsorbed state. all our approach is based on Pincus' theory for a stretched polymer.

\section{Introduction.}

The structure of linear polymers at interfaces has been under strong investigation these last few years. These studies started with the pioneering work of Hoeve, di Marzio and Peiser [1], Rubin [2] and Silberberg [3]. Mean field approach was refined by the Dutch group [4] these last few years. A different approach, linked to surface critical phenomena [5], was initiated by Lubensky and Rubin [6]. More recently, de Gennes and Pincus [7] reformulated a scaling theory that was originally developped and tested numerically by Binder, Eisenriegler and Kremer [8]. It was possible to define several surface concentration regimes with different conformations for the polymers $[9,10]$. It was found that when the surface is dilute, the adsorbed chain takes a pancake shape, and sticks to the adsorbing wall. When surface concentration increases, because of the excluded volume interaction between monomers, large loops develop in the solution $[10,11]$ and a concentration profile appears progressively.

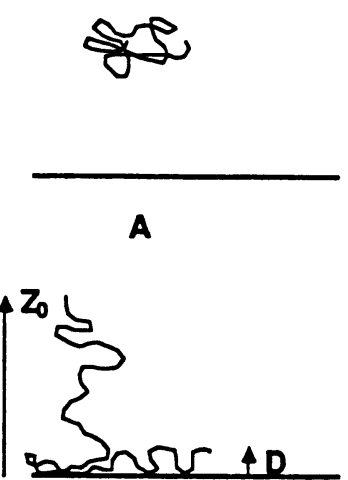

B

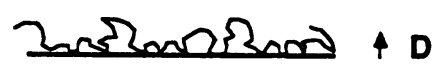

C

Fig. 1. - The different possible configurations of a chain: (a) isotropic, in the bulk when located at $z>Z_{0}$; (b) for $z<Z_{0}$, it is stretched. For $z \sim D$, it sticks to the surface and has a two dimensional extent (see text).

In the plateau regime, when the surface is saturated with polymers, the effect of these loops is to introduce a slowly decaying profile that extends to distances of the order of the radius of gyration of an isotropic, non adsorbed polymer. Although computer simulations $[8,14]$ and some neutron scattering [12] and reflectivity experiments [13] seem to support this power law decay of the profile, 
the situation is not clear, and agreement has not yet been reached among different teams for the plateau regime. It seems however that in the dilute surface regime, there is a strong belief that the polymers stick to the surface and adopt a pancake shape. In what follows, we would like to focus mainly on this case and to consider the relaxation process of such chain when the fixed end is loosened. This is related to an observation that was made by Eisenriegler $e t$ al. [8] who carried out detailed calculations of various quantities in the mean field case. Among others, they calculated the configurations of a chain with one end fixed at a distance $z$ from the surface. They showed that when $z$ is large enough, the chain adopts an isotropic conformation and does not feel the attraction by the surface. For smaller distances, the chain is attracted by the surface and stretches strongly. The cross-over distance $z_{0}$ between these two regimes depends on both the chain length $N$ and the monomer-surface interaction $\delta$. Relaxation of such chain when the constraint is released is also interesting to consider. This may be related to the extraction process of an adsorbed chain when one applies a force on one end. We will argue that such relaxation occurs by a succion of the stretched polymer onto the surface.

In the following, we will first recall the mean field results of Eisenriegler et al. Their generalisation to the case when excluded volume interactions are taken into account will be given in section 3. Section 4 is devoted to the relaxation time of a stretched chain. Finally, we discuss some possible experimental realizations of these results. Our scaling approach will follow closely Pincus' theory of stretched polymers.

\section{Mean field results.}

We consider a linear polymer chain made of $N$ units of length $l$ each. We assume that the chain is Gaussian and that there is no interactions between the monomers. Every monomer has an attractive potential with a surface. We will assume that the surface is impenetrable (such assumption is relevant for the self avoiding walk problem). This potential $W(z)$ is usually replaced by an effective interaction energy gain $\delta$ for a monomer on the surface. The equivalence between the problem of the configurations of a polymer and a magnetic problem in the presence of a surface was discussed by de Gennes [15] and by Eisenriegler $e t$ al [8]. We follow the latter approach here. In the continuous limit, $N \longrightarrow \infty$ limit, with $N l^{2}$ finite, the number $Z\left(\mathbf{r}, \mathbf{r}^{\prime}\right)$ of configurations of a chain with ends fixed respectively at $\mathbf{r}$ and $\mathbf{r}^{\prime}$ is

$$
Z\left(\mathbf{r}, \mathbf{r}^{\prime}\right)=\sum_{i} \mathrm{e}^{-E_{i} N l^{2}} \Psi_{\alpha}(\mathbf{r}) \Psi_{\alpha}^{*}\left(\mathbf{r}^{\prime}\right)
$$

where the $\Psi_{i}$ are the eigenfunctions of the Schrödinger equation

$$
[-\Delta+W(z)] \Psi_{i}=E_{i} \Psi_{i}
$$

For sufficiently low temperatures, equation (2) has bound states. By increasing the temperature, these bound states disappear. The disappearance of the last one corresponds to the adsorption temperature $T_{\mathrm{a}}$. We shall be interested in the vicinity of this threshold. In the continuous limit that we are considering, only the ground state with $E=0$ and eigenfunctions corresponding to vanishing energies contribute to the sum (1). In such a situation of ground state dominance, it was shown by de Gennes [16] that the solution of such equation is the same as for a Schrödinger equation without any potential but with the boundary condition

$$
\frac{1}{\Psi}[\partial \Psi / \partial z]_{z=0} \equiv \delta
$$


The latter logarithmic derivative defines an "extrapolation length" $D$ that is the characteristic length of the problem.

$$
D \sim \delta^{-1}
$$

When the chain is adsorbed, it adopts a pancake shape and is confined within a distance $D$ along the surface. It is then possible to calculate exactly $Z\left(\mathbf{r}, \mathbf{r}^{\prime}\right)$ and the number of configurations $Z(z)$ of a chain with one end fixed at a distance $z$ from the surface.

$$
Z(z)=\int_{0}^{\infty} Z\left(z, z^{\prime}\right) \mathrm{d} z^{\prime}
$$

This was calculated in reference [8] and we merely quote their results.

$$
\text { For } z \longrightarrow \infty, Z(z) \longrightarrow Z_{\text {bulk }} \sim 1
$$

where $Z_{\text {bulk }}$ is the partition function of a free chain, without any wall. Note that the normalisation is different from what is usually taken.

For smaller distances, it was found that

$$
Z(z)=1+2 \exp \left[\left(z_{0}-z\right) / D\right]
$$

with

$$
z_{0} \sim N \delta
$$

and $D$ given by equation (4).

Thus because of the occurence of bound states, two lengths appear in the problem, namely the width $D$ of the adsorbed polymer, and the capture distance $z_{0}$. Note that at the adsorption cross over, for $\delta N^{1 / 2} \sim 1$, all the various lengths merge with the isotropic radius of a free chain : $R \sim z_{0} \sim D$. Relation (6) allows us to calculate the force $F$ that has to be applied to the end to keep it at a fixed position.

$$
F=-\partial \operatorname{Ln} Z(z) / \partial z
$$

As noted in reference [8], apart from a region of width $D$ around $Z_{0}$, this force is

$$
F= \begin{cases}0 & \left(z>z_{0}\right) \\ \delta & \left(z<z_{0}\right)\end{cases}
$$

Thus the polymer does not feel the surface as long as it is located at distances larger than $z_{0}$. On the other hand, when one end of the chain comes at a distance equal to or smaller than $z_{0}$, the surface exerts a force $F$ that stretches it. In what follows, we will be concerned only with those polymers that are sufficiently close to the surface that they are stretched. It is possible to implement on the structure of these chains, following Pincus' theory of a stretched polymer. In the latter approach, one introduces tensile blobs made of $g$ monomers and with radius $\xi$ such that

$$
F \xi \sim k_{\mathrm{B}} T
$$

Such blobs are isotropic because the stretching energy is of the order of the thermal energy.

$$
\xi \sim g^{1 / 2}
$$


The stretched polymer is a linear string of blobs. Let us assume that the end of our chain is fixed at a distance $z_{0}$. Then we have

$$
z_{0} \sim \frac{N}{g} \xi
$$

Combining relations $(9 b)$ to $(12)$, we finally get

$$
\xi \sim F^{-1} \sim \delta^{-1}
$$

and

$$
z_{0} \sim N \delta
$$

It is interesting to note that the blob radius is of the same order than the width $D$ of the adsorbed polymer. When the end is fixed at a distance $z$ smaller than $z_{0}$, a relation similar to (12a) is valid, with $z$ replacing $z_{0}$. This implies that $M$ monomers are in the vicinity of the surface, within a distance $D$, because the applied force is still of the order of $\delta$, as found in (9b). Generalizing relation (12a), and solving, we get

$$
M \sim N-z \delta^{-1}
$$

\section{The self avoiding walk.}

The above results are easily generalized to the case when excluded volume interactions are present between the monomers. In what follows, we will assume the existence of a capture distance $Z_{0}$ and of a constant force $F$ applied to the polymer chain when it comes closer than $Z_{0}$ to the attractive surface. This generalization may be done using a scaling argument. In a good solvent the radius of a chain may be written in the following form:

$$
R=N^{3 / 5} f\left(\delta N^{3 / 5}\right)
$$

where we have used the Flory value for the excluded volume exponent [17], and approximated the cross-over exponent $[8,18,19]$ by $3 / 5$, for 3 dimensional systems. When the chain is adsorbed, it sticks to the surface and has a width $D$ that is obtained by assuming that $f(x)$ has a power law behavior. The exponent is readily determined by the condition that $D$ is independent of the length $N$. We find

$$
D \sim \delta^{-1}
$$

The adsorbed chain may be considered as a two dimensional chain made of blobs with size $D$. Because the solvent is good, each of these is swollen. Thus its radius $R_{\|}$in the adsorbing plane is [10]

$$
R_{\|} \sim N^{3 / 4} \delta^{1 / 4}
$$

Accepting the idea that was found in the mean field approximation, we assume the existence of a capture length $Z_{0}$ such that if the fixed end of the chain is farther than $Z_{0}$ the polymer does not feel the surface. If, on the contrary, the fixed end is closer than the capture length, the chain is stretched and may be viewed as a linear string of blobs with radius $D$. This implies that we also assume that the force $F$ needed to keep the end fixed at $z$ is constant whathever $z<Z_{0}$. Following Pincus' work on stretched polymers [20], this implies that the force is

$$
F \sim \xi^{-1} \sim D^{-1} \sim \delta
$$


where we have used relation (10). Because the tensile blobs are swollen, we have

$$
\xi \sim g^{3 / 5}
$$

Finally, the chain is stretched, so that we have, assuming that the end is fixed at $Z_{0}$

$$
Z_{0} \sim \frac{N}{g} \xi \sim N \delta^{2 / 3}
$$

In the same way as in the mean field approximation above, when the end is fixed at a distance $z$ smaller than $Z_{0}, M$ monomers are located within a distance $D$ from the surface, with

$$
M \sim N\left(1-\frac{z}{Z_{0}}\right)
$$

These results may be found in a Flory approximation [17], where we assume that every blob on the surface has an interaction energy of the order of the thermal energy... The elastic energy of a stretched string of $N-M$ units with length $z$ is

$$
F_{\mathrm{el}} \sim(z / l)^{1 /(1-\nu)}(N-M)^{\nu /(1-\nu)}
$$

Thus the total free energy is

$$
F=-M / g+F_{\mathrm{el}}
$$

Minimizing with respect to $M$ leads to relation (20).

One may check that at the adsorption cross-over, for $\delta N^{3 / 5} \sim 1$, the width of the adsorbed chain and the capture distance $Z_{0}$ merge with the isotropic radius of a free chain, relation (14) or (18). The existence of $Z_{0}$ and the stretched configuration may be checked by computer simulations. It might also be tested experimentally with a force machine such as used by Israelachvili et al. [23] and Klein [24]. In the latter case, one measures the onset of an attractive force when approaching from an attractive surface another one with grafted polymers. Note that in order that our approximation be valid, the density of grafted polymers has to be low enough.

\section{The relaxation times.}

Let us now consider the relaxation process of a polymer that had an end fixed at a distance $z$ equal to or less than $Z_{0}$, when this constraint is relaxed. As discussed above, it is initially attracted and stretched. Because it is not fixed any longer however, it relaxes the force and is adsorbed by a succion process. At the end of this step, the polymer is on the surface, with dimensions given by relations (4) and (17) normal to and in the plane respectively. The characteristic time for this second step is the relaxation time of a chain when an applied force is suddenly released. Such time was shown to be the same as the longest relaxation time of a polymer with an applied force. Following Pincus [21], it is possible to estimate these relaxation times. Two different characteristic times $T_{\mathrm{b}}$ and $T$ may be defined, respectively associated with the tensile blobs and the stretched polymer. Because we are considering a dilute solution, we will assume that hydrodynamic interactions are present when one considers the local motion of a blob. Then, the characteristic time $T_{\mathrm{b}}$ is a Zimm time [22]:

$$
T_{\mathrm{b}} \sim \xi^{3} \sim \delta^{-3}
$$


For larger distances, it was shown by Pincus that hydrodynamic interactions are screened, and that the larger times follow rather Rouse dynamics. Thus for the largest time, corresponding to the stretched chain, we find

$$
T \sim\left(\frac{N}{g}\right)^{2} T_{\mathrm{b}}
$$

and, using relations (17), (18) and (23), we find for a chain with an end initially fixed at position $z$

$$
T \sim D z^{2}
$$

Thus, the largest time, corresponding to a distance $z$ of the order of the capture ditance $Z_{0}$ is, using equation (19)

$$
T_{\mathrm{a}} \sim N^{2} \delta^{1 / 3}
$$

This corresponds to the adsorption time for a chain with one end initially fixed at a distance $Z_{0}$ that relaxes the applied force by sticking on the adsorbing plane. Note that this is larger than the characteristic time $\theta$ of an isotropic chain far from the surface. Again because the solution is dilute, the latter is a Zimm time

$$
\theta \sim N^{9 / 5}
$$

and thus

$$
\frac{T_{\mathrm{a}}}{\theta} \sim\left(\delta N^{\dot{3} / 5}\right)^{1 / 3}
$$

which is larger than unity for the adsorption case, $\delta N^{3 / 5} \gg 1$. Note however that both times merge at the adsorption cross-over.

\section{Discussion.}

For the single ideal chain adsorption, it was previously noted that in addition to the width $D$ of the adsorbed polymer, there exists a second characteristic length $Z_{0}$, corresponding to the capture distance by the attractive surface of a chain with an end fixed at a distance $z$. A polymer at a distance larger than $Z_{0}$ does not feel the attraction by the surface. A chain at a distance equal to or smaller than this distance is strongly attracted and adopts a stretched configuration. The applied force to keep the fixed end at the given distance was calculated for a Gaussian chain. Using scaling arguments, we generalized these notions to the case of a good solvent, when excluded volume interactions are present. It is possible to recover these results within a Flory approximation. An important result is the presence of two different lengths in the vicinity of the adsorption temperature. In addition to $D \sim \delta^{-1}$, where $\delta$ is the energy for a monomer on the surface and is proportional to the temperature difference $T-T_{\mathrm{a}}, T_{\mathrm{a}}$ being the adsorption temperature, we find $Z_{0} \sim N \delta^{2 / 3}$.

The relaxation of the polymer when the fixed end constraint is removed was also considered. It is initially strongly attracted by the surface and adopts a stretched configuration, corresponding to a constant applied force $F \sim \delta$. This is relaxed by a succion of the chain onto the adsorbing plane. The corresponding time $T$ is the longest characteristic time for the stretched chain and follows a Rouse dynamics. We find $T \sim N^{2} \delta^{1 / 3}$. All these dependences may be checked by computers simulations.

Finally we note that this time $T$ does not correspond to the adsorption time of a free chain from a solution, as we originally thought. Indeed, in the latter case, although the final, stretched, state has lower energy, the free chain has first to overcome an important energy barrier to reach it. This 
corresponds to the intermediate states when the chain is stretched and has no adsorbed monomers yet. Such fluctuation is a very rare event. Therefore a chain in a solution would rather diffuse until a distance of the order of its radius of gyration before being sucked onto the adsorbing plane. On the other hand, the relaxation process discussed above may be relevant for the extraction of a chain that is initially adsorbed, provided one pulls it from one end. A possible way to realize this is for instance by adding an electrical charge at one end and by applying an electric field.

\section{Acknowledgements.}

The authors are much indebted to L. Auvray, B. Farnoux and G. Jannink for various discussions and to one of our referees for correcting an important mistake in an earlier version of this paper.

\section{References}

[1] Hoeve C., Di Marzio E.A., Peyser P., J. Chem. Phys. 42 (1965) 2558.

[2] Rubin R., J. Chem. Phys. 43 (1966) 2392.

[3] Silberberg A.J., J. Chem. Phys. 46 (1967) 1105 ; 48 (1968) 2835.

[4] See for instance SCHEUTJENS J., Macromolecules at interfaces, $\mathrm{PhD}$ thesis, Wageningen, The Netherlands (1985).

[5] Binder K., HOHENBerg P.C., Phys. Rev. B6 (1972) 3461.

[6] LuBENSKY T.C., RuBIN M.H., Phys. Rev. B11 (1975) 5433 ; B12 (1975) 3885.

[7 PinCUS P., DE GENNES P.G., J. Phys. Lett. 44 (1983) 241.

[8] EISENRIEGLER E., BiNDER K., KREMER K., J. Chem. Phys. 77 (1982) 6296.

[9] BOUCHAUd E., DaOUd M., J. Phys. France 48 (1987) 1991.

[10] BOUCHAUD E., Thesis, Université Paris 11-Orsay (1988).

[11] DE Gennes P.G., C.R. Acad. Sci. Paris II 294 (1982) 1317.

[12] AUVRAY L., COTTON J.P., Macromolecules 20 (1987) 202

[13] Sun X., FarnouX B., Bouchaud E., LAPP A., DaOUd M., JanninK G., Europhys. Lett. 6 (1988) 207.

[14] BINDER K., Phase transitions and critical phenomena, Eds. C. Domb, J. Lebowitz (Academic Press) Vol. 8 (1983).

[15] DE GENNES P.G., J. Phys. France 37 (1976) 1445.

[16] DE Gennes P.G., Rep. Prog. Phys. 32 (1987) 187.

[17] DE GENNES P.G., Scaling concepts in polymer physics (Cornell university press) 1979.

[18] DIEHL H., DIETRICH S., Phys. Rev. B24 (1981) 2878.

[19] IsHinABE T., J. Chem. Phys. 76 (1982) $5589 ; 77$ (1983) 3170.

[20] PINCUS P., Macromolecules 9 (1976) 386.

[21] PINCUS P., Macromolecules 10 (1977) 210.

[22] DOI M., EDWARDS S.F., The theory of polymer dynamics (Clarendon press, Oxford) 1986.

[23] ISRAELACHVILI J.N., TIRRELl M., KIEIN J., AlMOG Y., Macromolecules 17 (1984) 205.

[24] KLEIN J., J. Chem. Soc., Faraday Trans. 79 (1983) 99. 